\title{
Accuracy of a New Hysteroscopic Method in the Assessment of Tubal Patency: Hysteroscopic Chromotubation
}

\author{
Burak YUCEL¹, Emine DEMIREL ${ }^{1}$, Sefa KELEKCI², Kerem Doga SECKIN ${ }^{1}$, Osama SHAWKI ${ }^{3}$ \\ Istanbul, Turkey
}

\section{ABSTRACT}

OBJECTIVE: Our aim was to determine the diagnostic accuracy of office hysteroscopy with hysteroscopic chromotubation findings about tubal patency in comparison with the laparoscopic chromopertubation.

STUDY DESIGN: Thirty-four healthy, fertile women who had tubal ligation for contraception and thirty women with unexplained infertility were included to the study. Both fallopian tubes of patients were evaluated for tubal patency with hysteroscopic chromotubation and laparoscopic chromopertubation. Finally, we calculated the accuracy of hysteroscopic chromotubation in diagnosing tubal patency.

RESULTS: Hysteroscopic chromotubation was positive in 100 patients, while 91 of these patients had patent tubes and nine had non-patent tubes in laparoscopy. Twenty-eight woman had negative hysteroscopic chromotubation. Thirteen of 28 patients with negative hysteroscopic chromotubation were confirmed with laparoscopically, however remaining 15 patients had patent tubes in laparoscopy. The sensitivity, specificity, positive predictive and negative predictive values for hysteroscopic chromotubation were $85.85 \%, 59.09 \%, 91.00 \%, 46.43 \%$, consequently.

CONCLUSION: Hysteroscopic chromotubation could give the chance to practitioners to assess tubal patency during hysteroscopy with high sensitivity and positive predictive value. Conclusions of our study needs to be confirmed with large cohorts from further studies.

Keywords: Hysteroscopy, Tubal patency, Choromotubation, Chromopertubation, Methylene blue

Gynecol Obstet Reprod Med 2018;24(2):82-86

\section{Introduction}

The Fallopian tubes are highly specialized and serve a number of essential functions in the reproductive process. The

${ }^{1}$ Clinic of Obstetrics and Gynecology University of Health Sciences Kanuni Sultan Suleyman Research and Training Hospital, Istanbul

2 Department of Obstetrics and Gynecology, Katip Celebi University School of Medicine, Izmir

${ }^{3}$ Department of Obstetrics and Gynecology, Cairo University School of Medicine, Cairo Egypt

Address of Correspondence: Burak Yucel

University of Health Sciences Kanuni

Sultan Suleyman Research and Training

Hospital 34303 Altinsehir

Küçükçekmece, Istanbul, Turkey

drburakyucel@gmail.com

Submitted for Publication: $\quad$ 17.03.2017

Accepted for Publication: $\quad$ 07.01.2018

\begin{tabular}{|c|c|}
\hline & Access this article online \\
\hline $\begin{array}{c}\text { Quick Response Code: } \\
\text { Website: www.gorm.com.tr } \\
\text { e-mail: info@gorm.com.tr }\end{array}$ & \begin{tabular}{c} 
DOI:10.21613/GORM.2017.692 \\
\cline { 2 - 3 }
\end{tabular} \\
\hline
\end{tabular}

How to cite this article: Yucel B. Demirel E. Kelekci S. Seckin KD. Shawki O. Accuracy of a New Hysteroscopic Method in the Assessment of Tubal Patency: Hysteroscopic Chromotubation. Gynecol Obstet Reprod Med $2018 ; 24(2): 82-86$ functional Fallopian tubes capture ovulated ova and transport sperm and embryos (1). Tubal pathology is among the most common causes of female infertility and the primary diagnosis in approximately $30-35 \%$ of infertile women (2).

The assessment of tubal patency (TP) is the one of the first step of the infertility evaluation. Hysterosalpingography (HSG) has been used to image the uterine cavity and to reveal the internal architecture of the tubal lumen since decades. It has some advantages, including the lack of need for anesthesia, relative speed with which the procedure is completed, and a potential therapeutic effect with oil soluble contrast media. A potential limitation of HSG is cornual spasm where uterine contractions that transiently close the interstitial segment and prevent distal perfusion (3). It has been determined that HSG may give a 10 percent to 20 percent false positive diagnosis of tubal obstruction. The other disadvantages are uncomfortable or painful procedure, some radiation exposure, and risk of infectious complications that can further impair fertility (4). Laparoscopy (LPS) provides both a panoramic view of the pelvic reproductive anatomy and a magnified view of the uterine, ovarian, tubal, and peritoneal surfaces. Laparoscopic chromopertubation (LCT) (introducing methylene blue into the uterine cavity being injected through the cervical canal by a cannula during the procedure and then observation as it comes out of the ends of the tubes into the peritoneal cavity) 
is considered to be the gold standard for TP (5). However, LPS is an invasive surgical procedure, requires general anesthesia, provides no information regarding the uterine cavity and involves the usual risks of surgery (6).

Because of some disadvantages of HSG and LPS, alternative methods have been developed. Hysterosalpingo contrast sonography (HyCoSy) that involves the installation of an echogenic contrast medium into the uterine cavity through a trans-cervical catheter and followed by transvaginal sonography with or without Doppler ultrasonography (USG), has been compared favorably with HSG in the literature and has received growing attention as a minimally invasive screening test (7). In recent years, office hysteroscopy (HYS) became the gold standard for evaluating the uterine cavity in infertility work-up (8). However, it is insufficient to investigate TP or tubal anatomy. It has been defined that HYS combined with ultrasonography can be used in the evaluation of TP with an additional ultrasonographic examination (5). In this method, detecting of the distension fluid in the cul-de-sac by USG was used as a test of TP.

We hypothesized that the observation of highly concentrated methylene blue transport or non-transport from uterine cavity to the tubes during HYS could identify TP in fertile and infertile women. The test was defined as hysteroscopic chromotubation (HCT) in the present study. We aimed to determine the diagnostic accuracy of office HSC with HCT findings about TP in comparison with the LPS, taking as a gold standard.

\section{Material and Method}

The population of this prospective cohort study consisted of both fertile women planned for LPS for tubal ligation and infertile women planned for LPS as a part of infertility workup at Gynecology and Obstetrics Department. The study was conducted between May 2013 and February 2015. Local Ethics Committee (2013/35) approved the study protocol and it was in agreement with the Declaration of Helsinki for Medical Research involving Human Subjects. Explanation and notification of the procedure and its aim, were done for all patients and written consent was taken.

\section{Procedure}

Office HSC procedures were performed without any anesthesia or sedation just before laparoscopies in the operating theatre. They were performed during the late proliferative phase (Day 11th to 14th of menstrual cycle). Continuous-flow hysteroscope with 30 degrees' oblique optic telescope, size with an outer diameter of $5 \mathrm{~mm}$, was used. Vaginoscopic approach were preferred without using a speculum and cervical dilatation. Contours of uterine cavity and tubal ostia were viewed. We used a diagnostic sheath with inflow and outflow channel. When we achieved good panoramic view, and then we closed the inflow and slowly injected highly concentrated
$(\% 10)$ methylene blue from inflow channel. Hysteroscope was moved closer to each side of tubal ostia (approximately one or one and a half centimeter to tubal ostium) to better observe the rhythmic movements of tubal ostium and the flow of the dye via ostium. The passage of fluid was visible by reducing the pressure in the uterus. Highly concentrated methylene blue does not mix immediately with the distending media (Normal saline with $0.9 \%$ of $\mathrm{NaCl}, 308 \mathrm{mOsm} / \mathrm{L}$ ) and therefore it can be observed in a nice flowing way. If rhythmic movements of tubal ostium and transport of highly concentrated methylene blue from uterine cavity to tubal ostium and tube were observed, the result of the test (hysteroscopic chromotubation) was defined as positive (Figure 1a, b, c); if not, the test was defined as negative (Figure 1d). Each tubal ostium was observed at least up to three minutes to assess the transport of the dye. $\mathrm{S}$ After office HSC ended, uterine manipulator was inserted after the induction of anesthesia just before the laparoscopic trocar insertion. Subsequently, TP was evaluated for both tubes laparoscopically, giving methylene blue from uterine manipulator in both the infertile and the fertile patients (laparoscopic chromopertubation). Finally, bilateral tubal ligation was performed with unipolar coagulation and diving by laparoscopic scissor in the fertile group.

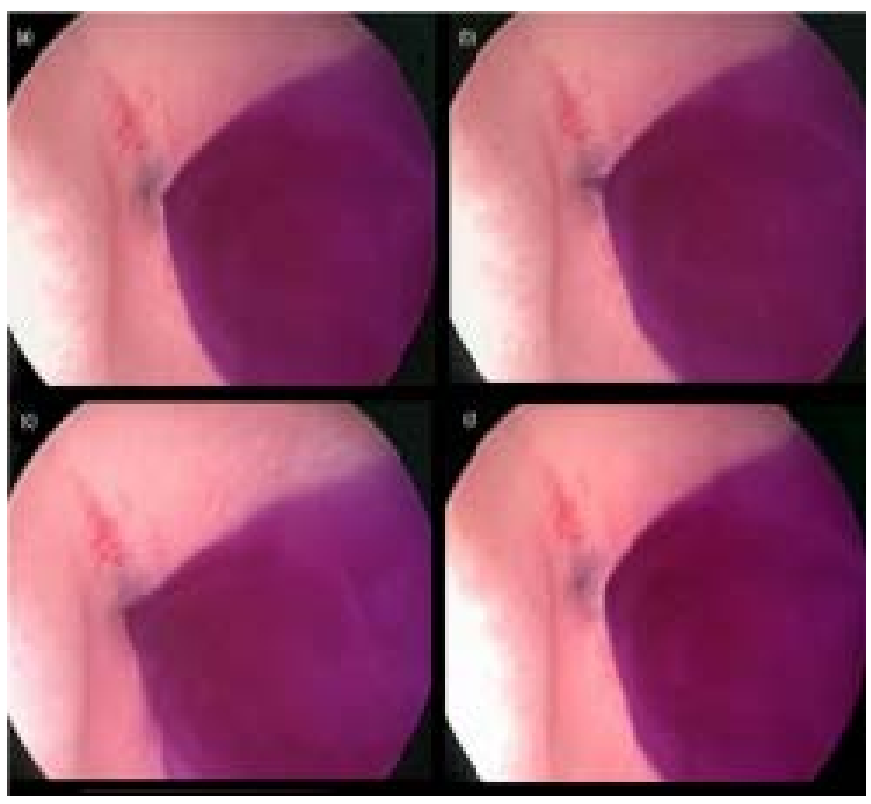

Figure 1: Hysteroscopic chromotubation, the observation of the flow of the highly concentrated methylene blue via tubal ostium (test positive) with an interval of one second (a), (b), (c). View of negative test (Where there was no passage of the highly concentrated methylene blue)

\section{Statistical analysis}

The results of HCT and LCT were evaluated for right and left tubes, separately. Data analysis was performed using SPSS Statistics for Macintosh version 20.0 (IBM Corporation, Armonk, NY, USA). Quantitative data are expressed as mean \pm standard deviation (SD). Categorical data are given as number (n) and percentage (\%). After the related tables were drawn for the tests, the accuracy of HCT in diagnosing TP, the 
sensitivity, the specificity, positive predictive value (PPV) and negative predictive value (NPV) were calculated.

\section{Results}

Totally sixty-four women (34 fertile women who had tubal ligation for contraception and 30 women with unexplained infertility) were included to the study. The mean age of fertile women was $35.71 \pm 3.83$, the mean age of infertile women was $29.03 \pm 3.11$, and the mean age of the whole study group was $32.58 \pm 4.84$. The mean values of body mass index for fertile and infertile women were $23.64 \pm 2.38$ and $23.70 \pm 3.81$, respectively. The mean value of body mass index of whole study group was $23.67 \pm 3.19$.

One hundred and twenty-eight tubes were determined with HCT and LCT. Results of the tests in diagnosing TP showed in table 1. The accuracy of HCT in diagnosing TP has been given in table 2 .

\section{Discussion}

Hysteroscopy is considered to be the gold standard to diagnose intrauterine pathology. Office HYS is being used increasingly in infertility work-up when TP is not an issue (9). Assessment of TP is not a part of routine hysteroscopic procedure. Adding evaluation of TP to routine HYS could provide several advantages. Our study showed that HCT could give useful information about TP with high sensitivity and moderate specificity compared to LCT.

Owing to its noninvasive nature and low cost, HSG is widely used as a first-line approach to assess TP; however, compared with LPS as gold standard, it is associated with sensitivity of $72 \%$ to $85 \%$ and specificity of $68 \%$ to $89 \%$ in diag- nosing TP (10). HSG also has a low sensitivity in diagnosis of intrauterine lesions, therefore it must be supplemented by HYS. In addition, HSG has disadvantages, including exposure to X-rays, radio-contrast material. Furthermore, it is dependent on an experienced radiologist. Another potential limitation of HSG is tubal spasm. (11). In our study; when we compared to LPS, chromotubation during HYS had similar sensitivity and specificity values with HSG in diagnosing TP. HYS also offers several advantages over HSG, namely that it allows the endometrial cavity to be visualized perfectly, simultaneously treats the pathology, and is not dependent on an experienced radiologist (1). In our opinion, observation of tubal ostia directly and high intrauterine pressures during procedure compared to HSG, provides an advantage in preventing false positive results due to tubal spasm.

Alternative methods to HSG have been developed for many years. With advances in ultrasound imaging, saline contrast sonography (SIS) and HyCoSy are recommended to replace HSG for the evaluation of the uterine cavity and TP in many centers worldwide (1). Rezk et al. evaluated the performance of SIS in diagnosis of TP compared to HSG: The sensitivity and negative predictive value of SIS were superior (12). Several studies cite HyCoSy's reliability, tolerability, rapidity, cost-effectiveness, its ability to provide information about the endometrial cavity and its potential advantage as a simple outpatient procedure: Lanzani et al. found HyCoSy to had a higher predictive value as a screening test than HSG (13). Hamed et al. found a $65 \%$ to $85 \%$ concordance rate between the results obtained by HyCoSy and those obtained by LCT (14). A meta-analysis of findings for 1007 women found a concordance rate of $83 \%$ between the HyCoSy results and those of either HSG or LCT (15). In contrast, disadvantages of HyCoSy in diagnosis TP has been also described. These in-

Table 1: Results of HCT and LCT in diagnosing tubal patency ${ }^{*}$

\begin{tabular}{lccc}
\hline & $\begin{array}{c}\text { Positive LCT } \\
\text { (patent tube) }\end{array}$ & $\begin{array}{c}\text { Negative LCT } \\
\text { (Not-patent tube) }\end{array}$ & Total \\
\hline Positive HCT (Patent tube) & 91 & 9 & 100 \\
Negative HCT (Not-patent tube) & 15 & 13 & 28 \\
Total & 106 & 22 & 128 \\
\hline
\end{tabular}

${ }^{*}$ All numbers refer to number of uterine tubes. HCT: Hysteroscopic chromotubation, LCT: Laparoscopic chromopertubation

Table 2: The accuracy of hysteroscopic chromotubation in diagnosing tubal patency

\begin{tabular}{lcc}
\hline & Value & 95\%Confidence interval \\
\hline Sensitivity & $85.85 \%$ & $77.74 \%$ to $91.86 \%$ \\
Specificity & $59.09 \%$ & $36.35 \%$ to $79.29 \%$ \\
Positive likelihood ratio & 2.10 & 1.26 to 3.49 \\
Negative likelihood ratio & 0.24 & 0.13 to 0.43 \\
Positive predictive value & $91.00 \%$ & $85.88 \%$ to $94.38 \%$ \\
Negative predictive value & $46.43 \%$ & $32.59 \%$ to $60.84 \%$ \\
\hline
\end{tabular}


clude potential causes for false interpretation of HyCoSy findings: observed echogenic flow in one segment of the tube without confirmation of distal flow over the adjacent ovary, presence of a tubal fistula where free tubal passage may mimic flow from the fimbria, and conversely, false occluded findings may be secondary to tubal spasm (1).

The indication of routine HYS for all infertile women is still a matter of controversy (16). HYS is the gold standard method for evaluation of intracavitary lesions; nevertheless, HYS is not a method to investigate TP (9). Several studies suggested that HYS could be used in evaluation of TP if combined with USG $(5,17)$. In this method, the tube is considered patent when the turbulence of the contrast is visualized on the side or in the Douglas pouch. Allam et al. found that the ultrasound saline contrast method for the assessment of the TP in comparison to LCT showed a sensitivity of $92.8 \%$, specificity of $50 \%$, positive predictive value of $92.8 \%$, negative predictive value of $50 \%$ (9). Examination of TP by the echogenic solution (Echovist-200) yielded a better specificity and positive predictive values. A meta-analysis by Holz et al. authenticated that saline HyCoSy had a sensitivity of $76.1 \%$ and a specificity of $79.4 \%$, with a positive predictive value of $71.4 \%$ (15). These results were comparable with the results of our study. Despite high specificity and positive predictive values, this method has several major limitations. Ultrasound images and their interpretation are subject to the experience and expertise of the practitioner. The investigation of TP is difficult to achieve, as the normal tubes are is poor sonic reflectors devoid of the defined interfaces that produce clear organ outlines (18). Additionally, this method cannot ensure bilateral tubal blockage in case of negative test due to the inadequate bilateral evaluation of tubes. In this condition, another method of TP assessment should be offered (1). HCT used in our study provides the assessment of the tubes separately. In our opinion, this is the major advantage of present technique superior to this method.

The major limitation of our technique includes the lack of the observation of the flow of the high concentrated methylene blue in the distal segment of the tube over the adjacent ovary. The relatively low sensitivity (59.09\%) of HCT that found in our study might be linked to this limitation. Other limitation of our study could be a relatively small sample of patients. Its prospective design and being the first study that discussing the accuracy of tubal chromotubation by HYS in the assessment of TP, are the major powers of our study.

In conclusion, according to analysis of our data; HCT has high sensitivity and moderate specificity values in assessment of TP compared to LCT. Adding HCT to routine office HYS, could give the chance to practitioners to assess TP without subjecting the patient to multiple procedures. It is also radiation free, minimally invasive, uses contrast material that is non-allergenic and does not require anesthesia. Conclusions of our study needs to be confirmed with large cohorts from further studies.

: Acknowledgement: The authors wish to thank Asst. Prof. Şafak Yücel who assisted in the proof-reading of the article. Conflict of interest: The authors declare no conflict of interest. Funding: None.

\section{References}

1. Saunders RD, Shwayder JM, Nakajima ST. Current methods of tubal patency assessment. Fertil Steril 2011;95 (7):2171-9

2. Develioğlu O, Özyürek E, Sadıkoğlu Y, Sadıkoğlu G, Tuncel E. Infertile women with bilateral obstructed tubes: (A presentation of 7 cases). Gynecol Obstet Reprod Med 2006;12(3):186-8.

3. Nugent D, Watson AJ, Killick SR, Balen AH, Rutherford AJ. A randomized controlled trial of tubal flushing with lipiodol for unexplained infertility. Fertil Steril 2002; 77(1):173-5.

4. Balsak D, Uysal F, Sadik S, Guler A, Tinar S, Taskin O. Comparison of hysterosalpingography and transvaginal hydrolaparoscopy in patients with tubal factor infertility: a prospective cohort study. Wideochir Inne Tech Maloinwazyjne 2014;9(2):190-5.

5. Habibaj J, Kosova H, Bilali S, Bilali V, Qama D. Comparison between transvaginal sonography after diagnostic hysteroscopy and laparoscopic chromopertubation for the assessment of tubal patency in infertile women. $\mathrm{J}$ Clin Ultras 2012;40(2):68-73.

6. Ngowa JD, Kasia JM, Georges NT, Nkongo V, Sone C, Fongang E. Comparison of hysterosalpingograms with laparoscopy in the diagnostic of tubal factor of female infertility at the Yaounde General Hospital, Cameroon. Pan Afr Med J 2015;22:264.

7. Helpman L, Wolman I. Hysterosalpingo contrast sonography for the evaluation of the tubal factor in infertility investigation: Review of literature. Rev Gynaecol Prac 2003;3(4):171-6.

8. Torok P. Use of hysteroscopy at the office in gynaecological practice. Orv Hetil 2014;155(40):1589-97.

9. Allam IS, Rashed AM, Sweedan KH, Bishry GAE, Ahmed WE. Role of hysteroscopy in the evaluation of tubal patency in infertile women. Mid East Fertil Soc J 2014;19:215-20.

10. Broeze KA, Opmeer BC, Van Geloven N, Coppus SF, Collins JA, Den Hartog JE, et al. Are patient characteristics associated with the accuracy of hysterosalpingography in diagnosing tubal pathology? An individual patient data meta-analysis. Hum Reprod Update 2011;17(3):293300 .

11. Perquin DA, Dorr PJ, de Craen AJ, Helmerhorst FM. Routine use of hysterosalpingography prior to la- 
paroscopy in the fertility workup: a multicentre randomized controlled trial. Hum Reprod 2006;21(5):1227-31.

12. Rezka M, Shawky M. The safety and acceptability of saline infusion sonography versus hysterosalpingography for evaluation of tubal patency in infertile women. Mid East Fertil Soc J 2015;20(2):108-13.

13. Lanzani C, Savasi V, Leone FP, Ratti M, Ferrazzi E. Twodimensional HyCoSy with contrast tuned imaging technology and a second-generation contrast media for the assessment of tubal patency in an infertility program. Fertil Steril 2009;92(3):1158-61.

14. Hamed HO, Shahin AY, Elsamman AM. Hysterosalpingocontrast sonography versus radiographic hysterosalpingography in the evaluation of tubal patency. Int $\mathrm{J}$ Gynaecol Obstet 2009;105(3):215-7.

15. Holz K, Becker R, Schurmann R. Ultrasound in the investigation of tubal patency. A meta-analysis of three com- parative studies of Echovist-200 including 1007 women. Zentralbl Gynakol 1997;119(8):366-73.

16. Hourvitz A, Ledee N, Gervaise A, Fernandez H, Frydman $\mathrm{R}$, Olivennes F. Should diagnostic hysteroscopy be a routine procedure during diagnostic laparoscopy in women with normal hysterosalpingography? Reprod Biomed 2002;4(3):256-60.

17. Youssef G, El-Sherbini M, El-Komy R, Tarek A. Combined transvaginal sonography and diagnostic hysteroscopy in the assessment of tubal patency in infertile women in correlation to laparoscopic chromopertubation. Evidence Based Women's Health Journal 2015;5(2):6771.

18. Mitri FF, Andronikou AD, Perpinyal S, Hofmeyr GJ, Sonnendecker EW. A clinical comparison of sonographic hydrotubation and hysterosalpingography. Bri J Obstet Gynaecol 1991;98(10):1031-6 